\title{
Effect of asthma and asthma medication on the prognosis of patients with COVID-19
}

\author{
Yong Jun Choi (1) ${ }^{1}$, Ju-Young Park $\mathbb{1}^{2}$, Hye Sun Lee $\mathbb{1}^{2}$, Jin Suh ${ }^{1}$,

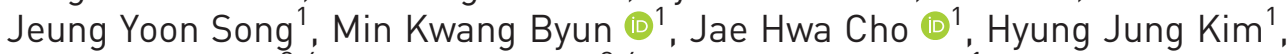 \\ Jae-Hyun Lee $\mathbb{1}^{3,4}$, Jung-Won Park ${ }^{3,4}$ and Hye Jung Park ${ }^{1}$
}

Affiliations: 'Dept of Internal Medicine, Gangnam Severance Hospital, Yonsei University College of Medicine, Seoul, Republic of Korea. ${ }^{2}$ Biostatistics Collaboration Unit, Yonsei University College of Medicine, Seoul, Republic of Korea. ${ }^{3}$ Division of Allergy and Immunology, Dept of Internal Medicine, Yonsei University College of Medicine, Seoul, Republic of Korea. ${ }^{4}$ Institute of Allergy, Yonsei University College of Medicine, Seoul, Republic of Korea.

Correspondence: Hye Jung Park, Division of Pulmonology, Dept of Internal Medicine, Yonsei University College of Medicine, Gangnam Severance Hospital, 211, Eonju-ro, Gangnam-gu, 06273 Seoul, Republic of Korea. E-mail: craft7820ayuhs.ac

@ERSpublications

Asthma increases the total medical cost burden and mortality rate associated with COVID-19. Asthma patients should not be concerned about using asthma medication during the COVID-19 pandemic, except for the use of oral short-acting $\boldsymbol{\beta}_{2}$-agonists. https://bit.ly/35L1yY8

Cite this article as: Choi YJ, Park J-Y, Lee HS, et al. Effect of asthma and asthma medication on the prognosis of patients with COVID-19. Eur Respir J 2021; 57: 2002226 [https://doi.org/10.1183/ 13993003.02226-2020].

\section{ABSTRACT}

Background: Coronavirus disease 2019 (COVID-19) has spread worldwide rapidly. However, the effects of asthma, asthma medication and asthma severity on the clinical outcomes of COVID-19 have not yet been established.

Methods: The study included 7590 de-identified patients, who were confirmed to have COVID-19 using the severe acute respiratory syndrome coronavirus 2 RNA-PCR tests conducted up to May 15, 2020; we used the linked-medical claims data provided by the Health Insurance Review and Assessment Service. Asthma and asthma severity (steps suggested by the Global Initiative for Asthma) were defined using the diagnostic code and history of asthma medication usage.

Results: Among 7590 COVID-19 patients, 218 (2.9\%) had underlying asthma. The total medical cost associated with COVID-19 patients with underlying asthma was significantly higher than that of other patients. Mortality rate for COVID-19 patients with underlying asthma (7.8\%) was significantly higher than that of other patients $(2.8 \% ; \mathrm{p}<0.001)$. However, asthma was not an independent risk factor for the clinical outcomes of COVID-19 after adjustment, nor did asthma medication use and asthma severity affect the clinical outcomes of COVID-19. However, use of oral short-acting $\beta_{2}$-agonists was an independent factor to increase the total medical cost burden. Patients with step 5 asthma showed significant prolonged duration of admission compared to those with step 1 asthma in both univariate and multivariate analysis.

Conclusions: Asthma led to poor outcomes of COVID-19; however, underlying asthma, use of asthma medication and asthma severity were not independent factors for poor clinical outcomes of COVID-19, generally.

This article has an editorial commentary: https://doi.org/10.1183/13993003.04451-2020

Received: 9 June 2020 | Accepted: 11 Sept 2020

Copyright OERS 2021. This version is distributed under the terms of the Creative Commons Attribution NonCommercial Licence 4.0 


\section{Introduction}

Coronavirus disease 2019 (COVID-19), named by the World Health Organization (WHO), has spread rapidly worldwide. The disease may manifest as mild upper respiratory symptoms, fever, fatigue, viral pneumonia, acute respiratory syndrome and death $[1,2]$. Coronavirus patients are usually infected through the respiratory tract, and the cause of death is mainly due to respiratory problems [3]. Asthma is a chronic airway inflammatory disease resulting in an imbalanced immunity of the airway [4]. Respiratory virus infections frequently exacerbate asthma $[5,6]$. We can assume that underlying asthma might affect the clinical outcomes of COVID-19 infection, since respiratory viruses broadly increase the risk of asthma exacerbation and death. In previous studies, underlying asthma is reported to account for $0.9-17 \%$ of hospitalised patients with COVID-19 $[1,7,8]$. However, the importance of underlying asthma is still overlooked in many COVID-19 studies, and there are currently no studies determining whether asthma is a risk factor for poor prognosis of COVID-19.

Inhaled medications, especially inhaled corticosteroids (ICS), are fundamental therapeutic options for asthma patients, and ICS are administered directly through the respiratory tract. Some asthma patients were concerned about the use of inhaled medications during the COVID-19 pandemic owing to the risk of respiratory infection through their inhaler device and suppression of the airway immunity system due to the use of ICS. The worldwide asthma guideline, Global Initiative for Asthma (GINA), recommends that asthma patients should continue taking their prescribed asthma medications, particularly ICS and oral corticosteroids, even during the COVID-19 pandemic period. However, studies supporting this recommendation have not been reported to date.

Korea has a national health insurance service (NHIS) that provides universal coverage for nearly all Korean citizens. All medical costs for COVID-19 in Korea have been covered by this service. The Health Insurance Review \& Assessment Service (HIRA) has extensive linked medical claims data (including inpatient and outpatient visits) of all the COVID-19 patients in Korea. In this study, we aimed to evaluate the effects of asthma and asthma medication use on the prognosis of COVID-19 using the national medical claims data for Korean patients. To the best of our knowledge, this is the first study to report on the association between asthma and COVID-19 using national cohort data.

\section{Materials and methods}

\section{COVID-19 patients and medical data}

The Ministry of Health and Welfare of Korea and HIRA shared with researchers the world's first de-identified COVID-19 nationwide patient medical claims data, covering the entire Korean population, via an online portal (accessible at https://hira-covid19.net). Among 234427 suspected COVID-19 patients, HIRA released the de-identified list of 7590 patients, who were confirmed to have COVID-19 by the severe acute respiratory syndrome coronavirus 2 RNA-PCR tests, conducted up to May 15, 2020 (figure 1). HIRA provided data linked to the COVID-19 patients' history of medical service use for the previous 3 years (using finalised claims data from January 2017 to May 2020).

\section{Underlying diseases and Charlson comorbidity index}

We included 17 underlying diseases (myocardial infarction, congestive heart failure, peripheral vascular disease, cerebrovascular disease, dementia, chronic pulmonary disease, rheumatologic disease, peptic ulcer disease, mild liver disease, diabetes without chronic complications, diabetes with chronic complications, hemiplegia or paraplegia, renal disease, any malignancy, including leukaemia and lymphoma, moderate or severe liver disease, metastatic solid tumour and AIDS/HIV infection), which are well-known underlying conditions that affect the mortality rate and are frequently used to calculate the Charlson comorbidity index (CCI) [9]. These underlying diseases were defined when a patient's claims data included a related diagnostic code at least once from January 1, 2019 to December 31, 2019. The diagnostic code was chosen according to the suggested guideline by the HIRA (accessible at https://opendata.hira.or.kr/op/opb/ selectRfrm.do?rfrmTpCd=\&searchCnd=\&searchWrd=\&sno=11200\&pageIndex=1). The CCI, which facilitates the prediction of disease prognosis and associated mortality, was calculated as described previously and according to the HIRA guidelines [9].

\section{Asthma, asthma medication and asthma severity}

An asthma diagnosis was determined when patients visited the hospital (at least once) due to asthma symptoms from January 2019 to December 2019. Furthermore, only patients who met the following criteria during the assessment period were regarded as having asthma: 1) International Classification of Diseases (ICD)-10 codes for asthma (J45 and J46) as primary diagnosis or first sub-diagnosis; and 2) prescription of asthma medications on at least two occasions during outpatient visits or prescription of asthma medication following an outpatient visit at least once and admission with treatment using systemic corticosteroids during the assessment period. This definition of asthma was established by the HIRA 


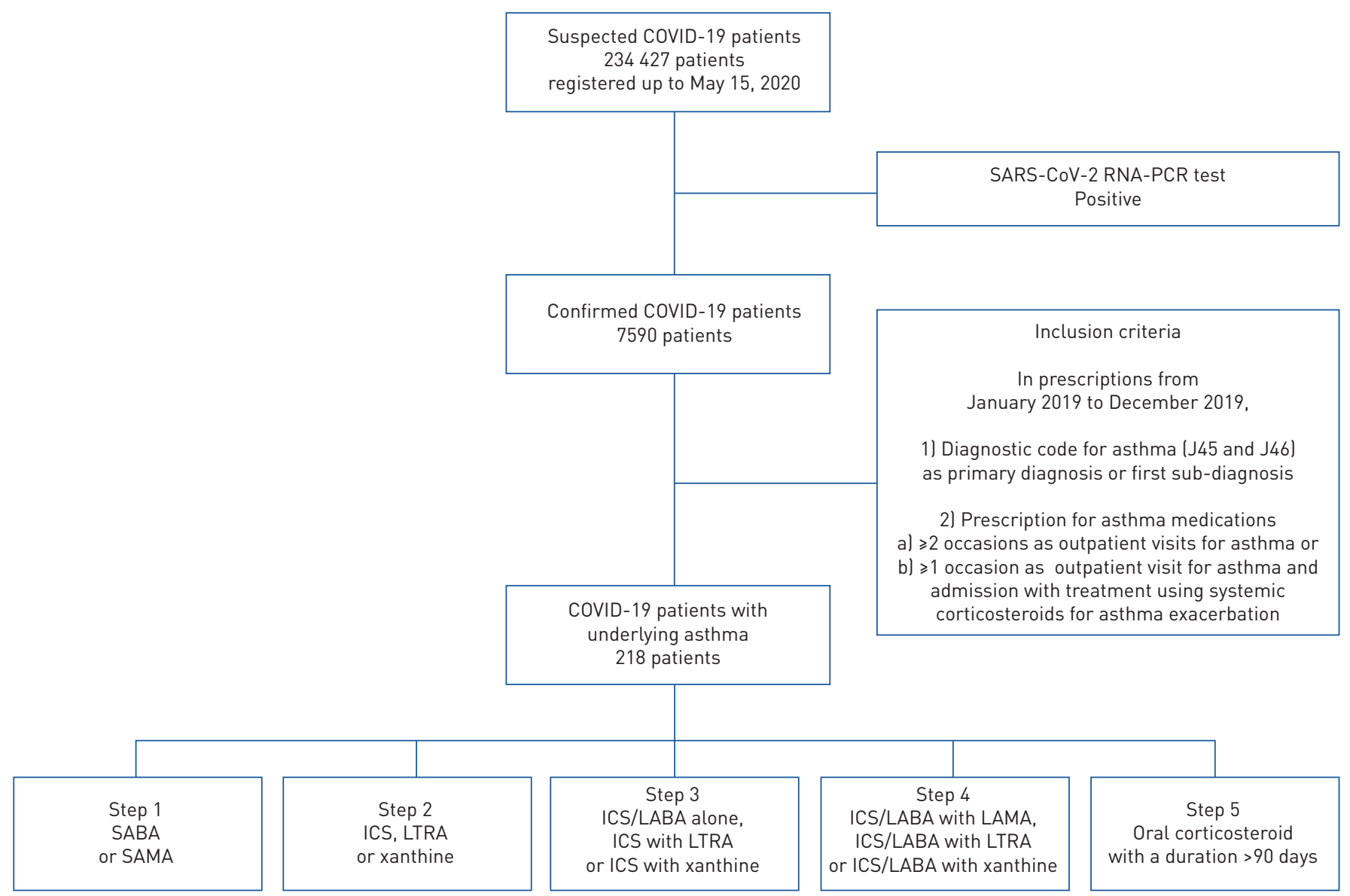

FIGURE 1 Overall study design. COVID-19: coronavirus disease 2019; SARS-CoV-2: severe acute respiratory syndrome coronavirus 2; SABA: short-acting $\beta_{2}$-agonist; SAMA: short-acting muscarinic antagonist; ICS: inhaled corticosteroid; LTRA: leukotriene receptor antagonist; LABA: long-acting $\beta_{2}$-agonist; LAMA: long-acting muscarinic antagonist.

committee according to the authoritative opinions of respiratory and allergy experts in Korea. Since then, this definition has been used by many clinical researchers, medical statisticians and the HIRA committee in Korea [10-12].

Asthma medication includes the following items: inhaled corticosteroids (ICS), inhaled long-acting $\beta_{2}$-agonists (LABA), oral LABA, patch LABA, systemic corticosteroids, leukotriene receptor antagonists (LTRA), xanthines, inhaled short-acting $\beta_{2}$-agonists (SABA), oral SABA and inhaled long-acting muscarinic antagonists (LAMA). In addition, we defined asthma medication usage if it occurred within the past year (from March 2019 to February 2020) and in the past 2 months (from January 2020 to February 2020). All asthmatic patients were classified based on the asthma medications used for the past year as follows. Step 1: SABA or short-acting muscarinic antagonist; step 2: ICS, LTRA or xanthine; step 3: ICS/LABA alone, ICS+LTRA or ICS+xanthine; step 4: ICS/LABA+LAMA, ICS/LABA+LTRA or ICS/ LABA+xanthine; step 5: oral corticosteroid with a duration $>90$ days following some modifications of the GINA treatment guidelines and a previous study [13].

\section{Clinical outcomes}

Mortality and admission to an intensive care unit (ICU), which are critical outcomes and represent severity of disease, and duration of admission and total medical costs, which represent the medical and social burden of disease, were evaluated. Reported ICD-10 diagnostic codes B342, B972, Z208, Z290, U18, U181, Z038, Z115, U071 and U072 during the treatment period for COVID-19 were considered as clinical outcomes. Total medical cost was defined as the sum of all medical costs for all the medical claims records for COVID-19 during the assessment period. All medical institutions must charge the total medical costs incurred by COVID-19 patients to the HIRA; and all the medical costs are covered by the Korean government. Therefore, all charged costs are consistent with the total cost of care of the patient. Duration of admission was defined as the duration from the admission date to the discharge date. 
Ethics

This study was approved by the institutional review board of Severance Hospital (Seoul, Republic of Korea; number 3-2020-0067). The requirement of obtaining informed consent was waived due to the retrospective nature of this study.

\section{Statistical analysis}

We calculated the CCI score to evaluate comorbidities and stratified the patients according to CCI scores of $0,1,2$ and $\geqslant 3$ points. In addition, we used logistic regression analysis to define risk factors for clinical outcomes and the results were adjusted for age, sex, underlying disease corresponding to each category in the CCI score and/or asthma medications. The multicollinearity between variables was evaluated by the variance inflation factor. The data were analysed using SAS Enterprise (version 6.1; SAS Institute, Cary, NC, USA). $\mathrm{p}<0.05$ was considered to indicate statistical significance.

\section{Results}

\section{Clinical characteristics and prognosis of COVID-19 patients}

Among 7590 COVID-19 patients, 218 (2.9\%) patients had underlying asthma. Age distribution was significantly different between the two groups $(\mathrm{p}<0.001)$. The CCI was significantly higher in COVID-19 patients with underlying asthma compared to that in COVID-19 patients without $(\mathrm{p}<0.001)$. Total medical cost in COVID-19 patients with underlying asthma (USD 5388.1) was higher than in others (USD 4261.0; $\mathrm{p}=0.003$ ). Duration of admission and prevalence of admission to ICU was not significantly different between the two groups. However, mortality rate in COVID-19 patients with underlying asthma $(7.8 \%)$ was significantly higher than others $(2.8 \%$; $<<0.001)$.

COVID-19 patients with underlying asthma were classified according to severity of asthma. There were no significant differences in age distribution, sex, mortality, admission to ICU and total medical cost between the steps. However, the CCI was significantly higher and duration of admission was significantly longer in patients with advanced steps (table 1).

\section{Usage of asthma medication in COVID-19 patients with underlying asthma}

In COVID-19 patients with underlying asthma, various asthma medications were used in the past year and in the past 2 months, respectively, as follows. LTRA (73.4\% and 66.5\%) and xanthine (50.5\% and $45.9 \%)$ were the most frequently used. Following them, ICS-LABA, inhaled SABA, oral LABA and ICS alone were also frequently prescribed (table 2).

\section{Significant factors for mortality}

Asthma was a significant factor for increased mortality rate in COVID-19 patients (OR 2.885, 95\% CI 1.726-4.822) in univariate analysis. However, it was not a significant factor after adjustment for age, sex and underlying conditions (OR 1.317, 95\% CI 0.708-2.451). In the univariate analysis, only usage of inhaled SABA or inhaled LAMA were risk factors for death. However, multivariate analysis showed that no asthma medications affected the mortality rate. The severity of asthma showed no significant association with mortality in both univariate and multivariate analysis (table 3 ).

\section{Significant factors for ICU admission}

Asthma was not a predictive factor for ICU admission in COVID-19 patients. Usage of oral SABA was a risk factor for ICU admission in univariate analysis; however, it was not a significant factor in multivariate analysis. In multivariate analysis, none of the asthma medications affected the risk of ICU admission. The severity of asthma was not significantly associated with ICU admission (table 4).

\section{Significant factors for admission duration}

Among the COVID-19 patients, asthma was not a significant factor affecting the duration of admission. Among the COVID-19 patients with underlying asthma, use of oral LABA in the past year and use of oral LABA in the past 2 months were protective factors for admission duration in the univariate analysis. However, they were not significant factors after adjustment. Patients with step 5 asthma showed significant prolonged admission duration than those with step 1 asthma in both univariate and multivariate analysis ( $\beta$ coefficient 19.583, 95\% CI 6.011-33.155 and $\beta$ coefficient $18.414,95 \%$ CI $4.031-32.796$, respectively) (table 5).

\section{Significant factors for total medical cost}

In COVID-19 patients, asthma was a significant factor in increasing total medical cost burden ( $\beta$ coefficient $1384449,95 \%$ CI 466632-2302266) in univariate analysis. However, after adjustment for age, sex and underlying conditions, it was not a significant factor ( $\beta$ coefficient 524590, 95\% CI -384769-1433949). 


\begin{tabular}{|c|c|c|c|c|c|c|c|c|c|}
\hline & \multirow{2}{*}{$\begin{array}{l}\text { COVID-19 patients without } \\
\text { underlying asthma }\end{array}$} & \multirow{2}{*}{$\begin{array}{l}\text { COVID-19 patients with } \\
\text { underlying asthma }\end{array}$} & \multirow[t]{2}{*}{ p-value } & \multicolumn{6}{|c|}{ COVID-19 patients with underlying asthma } \\
\hline & & & & Step 1 & Step 2 & Step 3 & Step 4 & Step 5 & p-value \\
\hline Age years & & & $<0.001^{* *}$ & & & & & & 0.058 \\
\hline $0-9$ & $62(0.8)$ & $20(9.2)$ & & $2(3.9)$ & $0(0.0)$ & $0(0.0)$ & $18(19.2)$ & $0(0.0)$ & \\
\hline $10-19$ & $343(4.7)$ & $6(2.8)$ & & $1(2.0)$ & $0(0.0)$ & $1(4.2)$ & $4(4.3)$ & $0(0.0)$ & \\
\hline $20-29$ & $1833(24.9)$ & $19(8.7)$ & & $8(15.7)$ & $3(6.7)$ & $3(12.5)$ & $5(5.3)$ & $0(0.0)$ & \\
\hline $30-39$ & $758(10.3)$ & $18(8.2)$ & & $4(7.8)$ & $3(6.7)$ & $1(4.2)$ & $10(10.6)$ & $0(0.0)$ & \\
\hline $40-49$ & 983 (13.3) & 25 (11.5) & & $7(13.7)$ & $5(11.1)$ & $4(16.7)$ & 8 (8.5) & $1(25.0)$ & \\
\hline $50-59$ & $1468(19.9)$ & $34(15.6)$ & & $10(19.6)$ & $6(13.3)$ & $5(20.8)$ & $13(13.8)$ & $0(0.0)$ & \\
\hline $60-69$ & 1020 (13.8) & $36(16.5)$ & & $7(13.7)$ & $12(26.7)$ & $4(16.7)$ & 13 (13.8) & $0(0.0)$ & \\
\hline$\geqslant 70$ & $846(11.5)$ & $60(27.5)$ & & $12(23.5)$ & $16(35.6)$ & $6(25.0)$ & 23 (24.5) & $3(75.0)$ & \\
\hline Sex & & & 0.393 & & & & & & 0.923 \\
\hline Male & $3000(40.7)$ & $95(43.6)$ & & $23(45.1)$ & $20(44.4)$ & $12(50.0)$ & $38(40.4)$ & $2(50.0)$ & \\
\hline Female & $4372(59.3)$ & $123(56.4)$ & & $28(54.9)$ & $25(55.6)$ & $12(50.0)$ & $56(59.6)$ & $2(50.0)$ & \\
\hline Charlson comorbidity index & & & $<0.001^{* *}$ & & & & & & $0.017^{*}$ \\
\hline 0 & 2029 (27.5) & $0(0.0)$ & & $0(0.0)$ & $0(0.0)$ & $0(0.0)$ & $0(0.0)$ & $0(0.0)$ & \\
\hline 1 & $1726(23.4)$ & $48(22.0)$ & & $12(23.5)$ & $3(6.7)$ & $5(20.8)$ & $28(29.8)$ & $0(0.0)$ & \\
\hline 2 & $1179(16.0)$ & 40 (18.4) & & $11(21.6)$ & $5(11.1)$ & $7(29.2)$ & $17(18.1)$ & $0(0.0)$ & \\
\hline$\geqslant 3$ & $2438(33.1)$ & $130(59.6)$ & & $28(54.9)$ & 37 (82.2) & $12(50.0)$ & $49(52.1)$ & $4(100)$ & \\
\hline \multicolumn{10}{|l|}{ Prognosis } \\
\hline Mortality & $210(2.8)$ & $17(7.8)$ & $<0.001 * *$ & $5(9.8)$ & $2(4.4)$ & $1(4.2)$ & $9(9.6)$ & $0(0.0)$ & 0.703 \\
\hline Admission to ICU & $208(2.8)$ & 7 (3.2) & 0.733 & $3(5.9)$ & $1(2.2)$ & $0(0.0)$ & 3 (3.2) & $0(0.0)$ & 0.691 \\
\hline Duration of admission days & $20.1 \pm 12.2$ & $21.0 \pm 13.5$ & 0.285 & $18.7 \pm 12.0$ & $23.7 \pm 15.7$ & $22.7 \pm 13.0$ & $19.9 \pm 12.6$ & $38.3 \pm 16.4$ & $0.026 *$ \\
\hline \multirow[t]{2}{*}{ Total medical cost ${ }^{\#}$ USD } & $4261.0 \pm 5354.0$ & $5388.1 \pm 10135.3$ & $0.003^{*}$ & 4403.5 & 6242.7 & 4694.6 & 5669.8 & 5868.4 & 0.912 \\
\hline & & & & \pm 4992.1 & \pm 9180.5 & \pm 3815.9 & \pm 13470.9 & \pm 2789.8 & \\
\hline Total & 7372 (97.1) & 218 (2.9) & & $51(23.4)$ & $45(20.6)$ & $24(11.0)$ & $94(43.1)$ & $4(1.8)$ & \\
\hline
\end{tabular}


TABLE 2 Use of asthma medication in coronavirus disease 2019 (COVID-19) patients with underlying asthma

Asthma medication use

\begin{tabular}{lcc} 
& In the past year & In the past 2 months \\
\hline ICS alone & $66(30.3)$ & $58(26.6)$ \\
ICS-LABA & $84(38.5)$ & $78(35.8)$ \\
Inhaled LABA & $0(0.0)$ & $0(0.0)$ \\
Oral LABA & $77(35.3)$ & $70(32.1)$ \\
Patch LABA & $41(18.8)$ & $36(16.5)$ \\
LTRA & $160(73.4)$ & $145(66.5)$ \\
Inhaled SABA & $83(38.1)$ & $75(34.4)$ \\
Oral SABA & $30(13.8)$ & $8(3.7)$ \\
Xanthine & $110(50.5)$ & $100(45.9)$ \\
Inhaled LAMA & $25(11.5)$ & $25(11.5)$
\end{tabular}

Data are presented as $n(\%)$. ICS: inhaled corticosteroid; LABA: long-acting $\beta_{2}$-agonist; LTRA: leukotriene receptor antagonist; SABA: short-acting $\beta_{2}$-agonist; LAMA: long-acting muscarinic antagonist.

TABLE 3 Significant factors associated with mortality

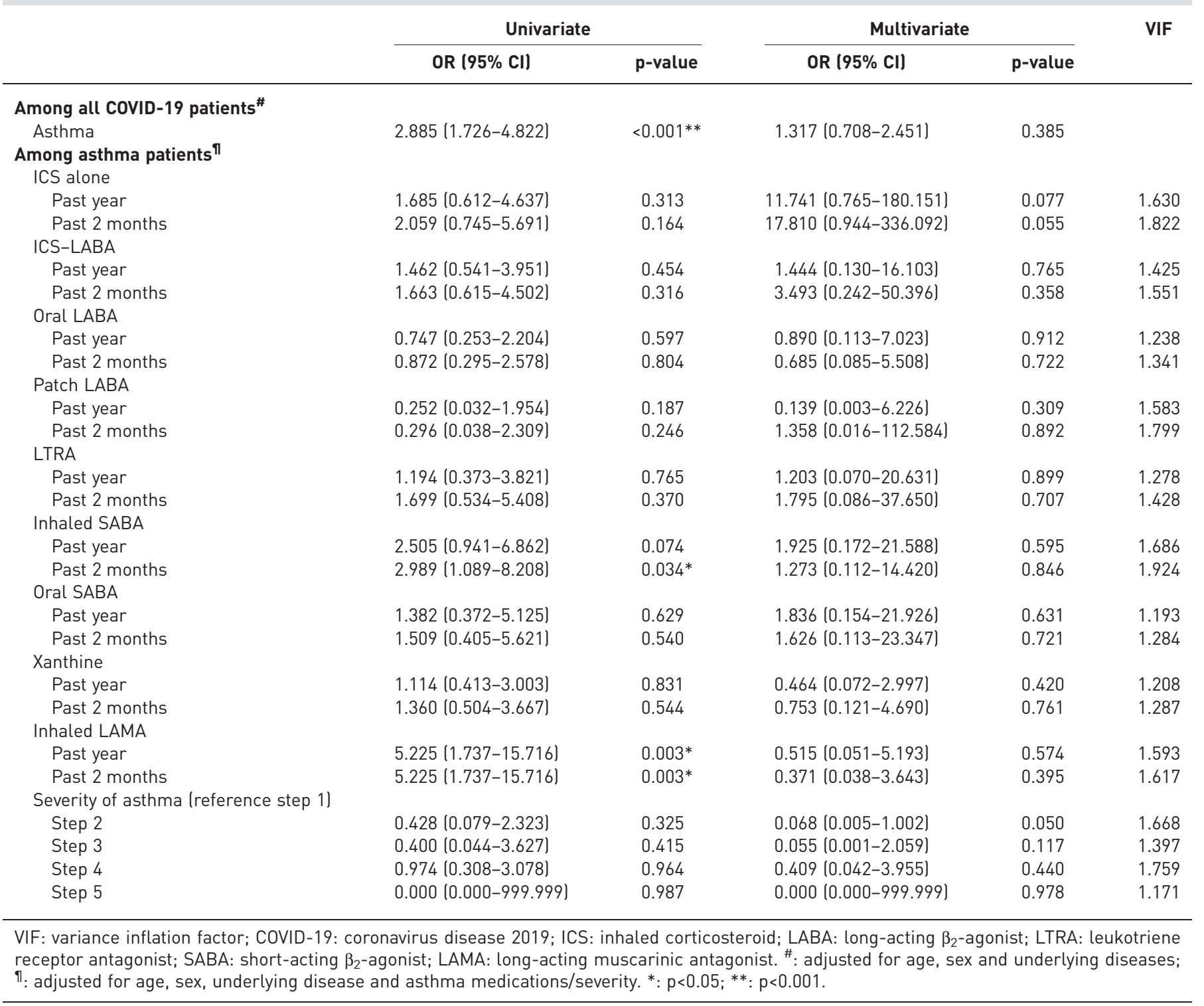


TABLE 4 Significant factors associated with intensive care unit admission

\begin{tabular}{|c|c|c|c|c|c|}
\hline & \multicolumn{2}{|c|}{ Univariate } & \multicolumn{2}{|c|}{ Multivariate } & \multirow[t]{2}{*}{ VIF } \\
\hline & OR $(95 \% \mathrm{CI})$ & p-value & OR $(95 \% \mathrm{CI})$ & p-value & \\
\hline \multicolumn{6}{|c|}{ Among all CoVID-19 patients" } \\
\hline \multicolumn{6}{|c|}{ Among asthma patients ${ }^{\pi}$} \\
\hline \multicolumn{6}{|c|}{ ICS alone } \\
\hline Past year & $0.919(0.174-4.861)$ & 0.921 & 3.802 (0.137-105.589) & 0.431 & 1.630 \\
\hline Past year & $0.629(0.119-3.320)$ & 0.585 & $0.384(0.029-5.036)$ & 0.466 & 1.425 \\
\hline Past 2 months & $0.711(0.135-3.751)$ & 0.687 & $0.503(0.046-5.451)$ & 0.572 & 1.551 \\
\hline \multicolumn{6}{|l|}{ Oral LABA } \\
\hline Past year & $0.725(0.137-3.830)$ & 0.705 & $0.373(0.021-6.561)$ & 0.500 & 1.238 \\
\hline Past 2 months & $0.841(0.159-4.446)$ & 0.839 & $0.254(0.010-6.487)$ & 0.407 & 1.341 \\
\hline \multicolumn{6}{|l|}{ Patch LABA } \\
\hline Past 2 months & $1.268(0.240-6.697)$ & 0.780 & $8.106(0.309-212.62)$ & 0.209 & 1.428 \\
\hline \multicolumn{6}{|l|}{ Inhaled SABA } \\
\hline Past year & $0.642(0.122-3.387)$ & 0.602 & $2.385(0.098-58.120)$ & 0.594 & 1.686 \\
\hline Past 2 months & $0.756(0.143-3.994)$ & 0.742 & $3.253(0.083-126.978)$ & 0.528 & 1.924 \\
\hline \multicolumn{6}{|l|}{ Oral SABA } \\
\hline Past year & $5.112(1.085-24.096)$ & $0.039 *$ & $4.484(0.317-63.484)$ & 0.267 & 1.193 \\
\hline Past 2 months & $5.581(1.180-26.402)$ & $0.030 *$ & $12.987(0.472-357.078)$ & 0.129 & 1.284 \\
\hline \multicolumn{6}{|l|}{ Xanthine } \\
\hline Past year & $1.321(0.289-6.045)$ & 0.720 & $0.319(0.019-5.444)$ & 0.430 & 1.208 \\
\hline Past 2 months & $1.597(0.349-7.312)$ & 0.546 & $0.564(0.028-11.527)$ & 0.710 & 1.287 \\
\hline \multicolumn{6}{|l|}{ Inhaled LAMA } \\
\hline Past year & 0.000 (0.000-999.999) & 0.968 & 0.000 (0.000-999.999) & 0.904 & 1.593 \\
\hline Past 2 months & $0.000(0.000-999.999)$ & 0.968 & $0.000(0.000-999.999)$ & 0.917 & 1.617 \\
\hline
\end{tabular}

VIF: variance inflation factor; COVID-19: coronavirus disease 2019; ICS: inhaled corticosteroid; LABA: long-acting $\beta_{2}$-agonist; LTRA: leukotriene receptor antagonist; SABA: short-acting $\beta_{2}$-agonist; LAMA: long-acting muscarinic antagonist. \#: adjusted for age, sex and underlying disease; ": adjusted for age, sex, underlying disease and asthma medications/severity. ${ }^{*}: p<0.05$.

In the COVID-19 patients with underlying asthma, usage of asthma medications did not affect the total medical cost, except for oral SABA. Usage of oral SABA in the past year ( $\beta$ coefficient $6258922,95 \%$ CI $1030068-11487776)$ and in the past 2 months ( $\beta$ coefficient 5861499, 95\% CI 264201-11458797) were significant factors to increase total medical cost burden, independently, in multivariate analysis. The severity of asthma was not significantly associated with total medical cost (table 6).

\section{Discussion}

This national study showed that COVID-19 patients with underlying asthma increase the medical cost burden and are more susceptible to mortality than COVID-19 patients without underlying asthma. However, asthma, asthma medication and asthma severity were not independent risk factors for clinical outcomes of COVID-19, after adjustment for confounding factors. Only usage of oral SABA increased the total medical cost burden independently. Patients with step 5 asthma showed longer duration of admission compared with those with step 1 asthma. This study's strength was that we included all the COVID-19 patients in Korea; however, the limitation was that we defined asthma patients based on the diagnosis code and usage of asthma medication.

The prevalence of asthma among the COVID-19 patients was $2.9 \%$ in this study, which is similar to that among the general South Korean population (1.6-2.2\%) [13-15]. Underlying asthma is reported to 
TABLE 5 Significant factors affecting the admission duration (days)

\begin{tabular}{|c|c|c|c|}
\hline \multicolumn{2}{|l|}{ Univariate } & \multicolumn{2}{|c|}{ Multivariate } \\
\hline$\beta$ coefficient $(95 \%$ CI $)$ & p-value & B coefficient $(95 \% \mathrm{CI})$ & p-value \\
\hline
\end{tabular}

\section{Among all CoVID-19 patients ${ }^{\#}$ \\ Asthma}

Among asthma patients"

ICS alone

Past year

Past 2 months

ICS-LABA

Past year

Past 2 months

Oral LABA

Past year

Past 2 months

Patch LABA

Past year

Past 2 months

LTRA

Past year

Past 2 months

Inhaled SABA

Past year

Past 2 months

Oral SABA

Past year

Past 2 months

Xanthine

Past year

Past 2 months

Inhaled LAMA

Past year

Past 2 months

Severity of asthma (reference step 1)

Step 2

Step 3

Step 4

Step 5
$0.947(-0.693-2.587)$

$-1.385(-5.306-2.537)$

$-2.805(-6.869-1.260)$

$0.611(-3.094-4.316)$

$0.055(-3.707-3.818)$

$-3.899(-7.636--0.162)$

$-4.248(-8.070--0.428)$

$0.520(-4.095-5.135)$

-0.948 (-5.804-3.908)

$-0.273(-4.354-3.809)$

$-1.812(-5.626-2.002)$

$-0.573(-4.286-3.141)$

$0.100(-3.697-3.896)$

$4.088(-1.119-9.295)$

$3.149(-2.225-8.523)$

$1.036(-2.569-4.641)$

$0.682(-2.937-4.300)$

$0.450(-5.210-6.111)$

$0.450(-5.210-6.111)$

$5.044(-0.301-10.390)$

$4.000(-2.470-10.470)$

$1.238(-3.308-5.783)$

19.583 (6.011-33.155)
0.258

$-0.342(-1.993-1.309)$

0.487

0.175

0.746

0.977

$0.041^{*}$

$0.030^{*}$

0.825

0.701

0.895

0.350

0.762

0.959

0.123

0.249

0.572

0.711

0.876

0.876

0.064

0.224

0.592

$0.005 *$
$-1.083(-6.096-3.929)$

$-3.579(-9.083-1.925)$

$-0.794(-5.220-3.631)$

$-1.994(-6.675-2.688)$

$-3.503(-7.703-0.696)$

$-3.744(-8.214-0.726)$

$4.516(-1.292-10.324)$

$3.302(-3.208-9.811)$

$0.662(-3.952-5.277)$

$-0.620(-5.184-3.944)$

$1.076(-3.748-5.900)$

$3.915(-1.347-9.177)$

$5.734(0.014-11.453)$

$4.893(-1.210-10.997)$

$-0.129(-4.095-3.836)$

$-0.225(-4.327-3.878)$

$-3.046(-10.192-4.099)$

$-2.431(-9.623-4.760)$

$4.565(-1.127-10.258)$

$2.739(-3.998-9.475)$

$0.976(-3.802-5.753)$

18.414 (4.031-32.796)
0.685

0.670

0.201

1.630

1.822

0.724

1.425

1.551

$0.102 \quad 1.238$

$0.100 \quad 1.341$

$0.127 \quad 1.583$

$0.318 \quad 1.799$

$0.777 \quad 1.278$

$0.789 \quad 1.428$

$0.661 \quad 1.686$

$0.144 \quad 1.924$

0.049* $\quad 1.193$

$0.115 \quad 1.284$

$0.949 \quad 1.208$

$0.914 \quad 1.287$

$0.402 \quad 1.593$

$0.506 \quad 1.617$

$0.115 \quad 1.668$

$0.424 \quad 1.397$

$0.688 \quad 1.759$

$0.012 * \quad 1.171$

VIF: variance inflation factor; COVID-19: coronavirus disease 2019; ICS: inhaled corticosteroid; LABA: long-acting $\beta_{2}$-agonist; LTRA: leukotriene receptor antagonist; SABA: short-acting $\beta_{2}$-agonist; LAMA: long-acting muscarinic antagonist. " : adjusted for age, sex and underlying disease;

ๆ: adjusted for age, sex, underlying disease and asthma medications/severity. *: $p<0.05$.

account for $0.9-17 \%$ of patients hospitalised with COVID-19 in other countries $[1,7,8]$. Some studies have reported that the prevalence of asthma in the COVID-19 population is lower than that expected in the general population [16, 17]; however, other studies showed that asthma was reported in up to $27 \%$ of cases [18]. These differences may have been caused by differences in region, race and policies as well as criteria for hospitalisation of COVID-19 patients. Additional large-scale cohort studies are required to evaluate the prevalence of asthma in patients with COVID-19 and establish whether asthma is a risk factor for COVID-19 infection.

In this study, underlying asthma was not an independent factor to predict poor clinical outcomes of COVID-19 after adjustment. Age distribution and CCI were significantly different between COVID-19 patients with and without asthma. In asthma patients, very young people (aged $<10$ years, 9.4\%) and old people (aged $\geqslant 60$ years, $42.5 \%$ ) were represented more frequently, compared to patients without asthma $(0.8 \%$ and $25.3 \%$, respectively). The negative effects of asthma on clinical outcomes in univariate analysis might be induced by these confounding effects (imbalanced distribution of age and CCI). After adjustment for basic clinical factors, the negative effects of asthma on COVID-19 disappeared. However, considering the mechanisms of asthma and COVID-19, we need further large-scale studies to confirm whether asthma is a significant factor for clinical outcomes in COVID-19. 
TABLE 6 Significant factors affecting the total medical cost (Korean Won)

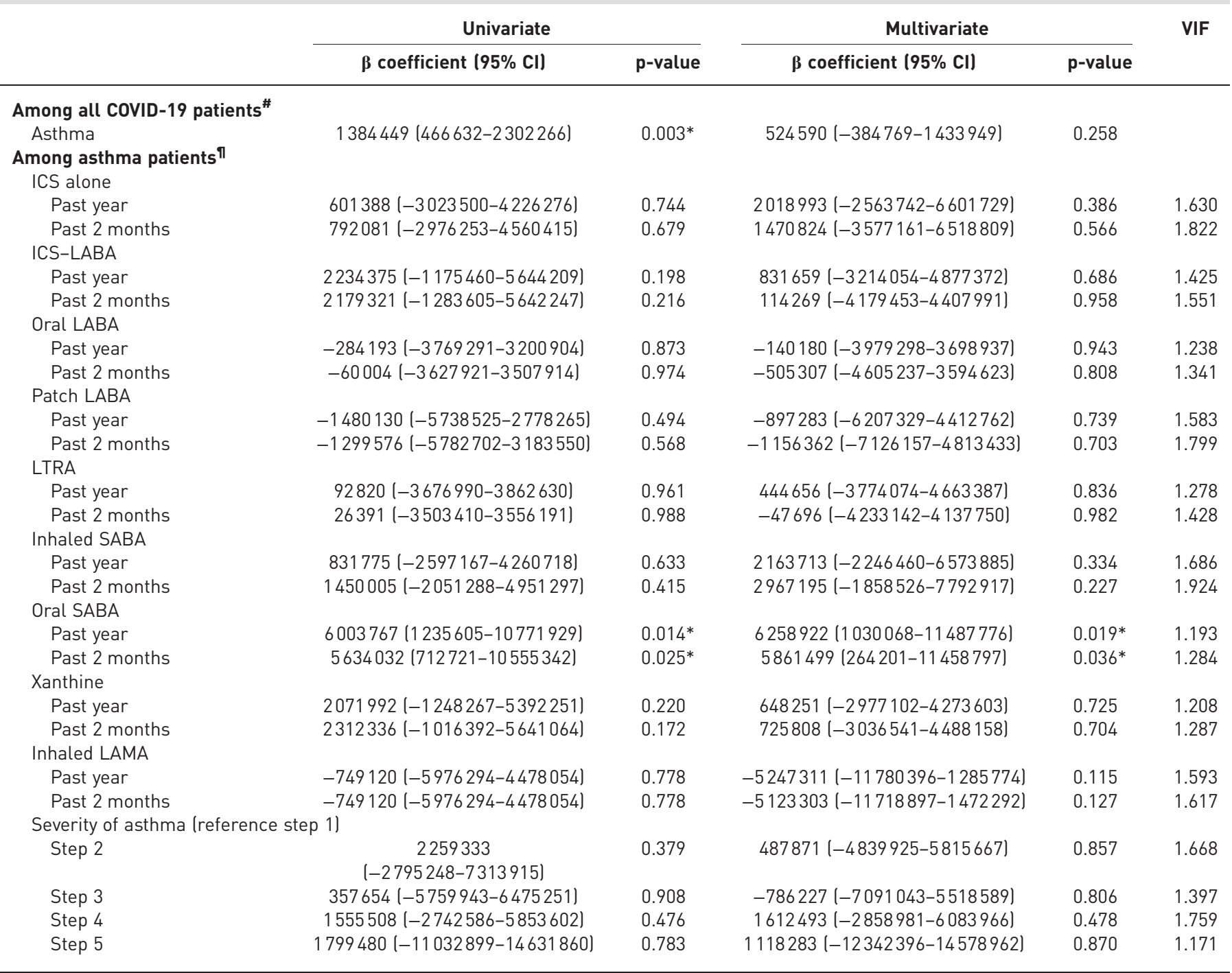

VIF: variance inflation factor; COVID-19: coronavirus disease 2019; ICS: inhaled corticosteroid; LABA: long-acting $\beta_{2}$-agonist; LTRA: leukotriene receptor antagonist; SABA: short-acting $\beta_{2}$-agonist; LAMA: long-acting muscarinic antagonist. \#: adjusted for age, sex and underlying diseases; ף: adjusted for age, sex, underlying disease and asthma medications/severity. ${ }^{*}$ : $p<0.05$.

Asthma medication did not affect the clinical outcomes in this study. The usage rate of asthma medication reported in this study is similar to that reported in general asthma patients, as described in a previous study [10]. GINA recommends that asthma patients continue taking their prescribed asthma medications during the COVID-19 pandemic period, because stopping asthma medication often leads to potentially dangerous exacerbation of asthma symptoms. Among asthma medications, steroids can have dual functions: blocking host hyperactive inflammation, including cytokine storming, and inducing the replication of coronavirus by blocking innate immunity $[19,20]$. Therefore, WHO guidance proposed that systemic corticosteroids should be carefully used only in selected cases. In addition, some recent Japanese studies have reported that ciclesonide, as one type of ICS, may have positive effects in treating COVID-19 $[21,22]$. However, independently, this study concludes that recent use of asthma medication did not affect clinical outcomes of COVID-19. In addition, the result of this study supports the GINA recommendation, indirectly. Further research is needed.

In this study, use of oral SABA increased the total medical cost burden independently. SABA, as a bronchodilator, relieves asthma symptoms; however, it has various side-effects, including tachycardia, tremors and hypokalaemia $[23,24]$. Recently, the GINA guidelines recommended that asthma patients should not receive SABA-only treatment, because SABA-only treatment increases the risk of severe 
exacerbation and asthma-related mortality, and addition of ICS significantly reduces the risk [25, 26]. Oral SABA has been prescribed frequently in very young or old people, as a substitute for inhaled SABA. However, it has a slower onset of action and the associated side-effects are noted more frequently compared to those for inhaled SABA. Therefore, oral SABA may lead to increased medical cost burden in COVID-19.

LAMA seems to be a significant factor for mortality in univariate analysis. This can be explained by the following reasons: 1) patients who use LAMA might have COPD features; 2) patients who use LAMA might have severe asthma because LAMA is recommended at step 4-5 based on the GINA guideline. After adjustment for other factors, LAMA was not a significant factor to predict mortality.

COVID-19 patients with underlying asthma showed a higher total medical cost and mortality rate compared to those without underlying asthma in this study. The airway epithelium is essential to control inflammatory and immune responses to allergens, viruses and pollutants, which invade through the respiratory tract [27]. However, dysfunction of the airway epithelium in asthma patients can lead to a weakened immune defence mechanism [27, 28]. Infection from a respiratory virus frequently induces asthma exacerbation, and it can also lead to poor clinical outcomes, and even death [5]. Autopsies of COVID-19 infected patients showed airway inflammation and diffuse alveolar damage in pathologic analysis [29], indicating that COVID-19 is also an airway inflammatory disease. Therefore, asthma might negatively affect clinical outcomes in COVID-19.

The severity of asthma was not related to mortality, ICU admission and total medical cost in this study, but it was significantly related with the duration of admission. However, because of limited evidence from previous studies and the small number of step 5 patients in this study, additional research is thought to be necessary to confirm these findings.

The strengths of this study are that we included almost all COVID-19 patients diagnosed in Korea, and we included an adequate number of asthma patients. The Korean NHIS includes all medical utilisation of almost all COVID-19 patients in Korea; and this minimises selection bias and collider bias. However, there are some limitations of this study. First, HIRA data includes only diagnostic codes and medication use; therefore, the definition of asthma was based on the diagnostic code and history of use of asthma medication, while asthma was originally diagnosed based on the clinical history and objective test results. Mild asthma patients with intermittent symptoms might have been excluded in this study. However, Korea has a national health insurance service that covers almost all Korean citizens. Asthma patients in Korea can visit hospitals more freely than in many other countries because of this insurance service. Hence, we considered that the number of patients excluded due to mild asthma would not be large. Second, we could not obtain the date of COVID-19 confirmation, real use of asthma medications and the type of medicinal ingredients and dose of medication. Third, we could not obtain important clinical and laboratory data, which can affect the clinical outcomes of COVID-19.

In conclusion, underlying asthma in COVID-19 patients is positively associated with medical cost burden and mortality. However, there was no evidence of an association between asthma, asthma medication or asthma severity and the clinical outcomes of COVID-19 after adjustment for confounding factors. Reflecting these results, clinicians can recommend that asthma patients continue using their asthma medication including ICS during the COVID-19 pandemic.

Acknowledgements: The authors appreciate the healthcare professionals dedicated to treating COVID-19 patients in Korea, and the Ministry of Health and Welfare and the Health Insurance Review and Assessment Service of Korea for sharing invaluable national health insurance claims data in a prompt manner.

Author contributions: Y.J. Choi analysed and interpreted the data, drafted and revised the article, and approved the final version of this manuscript for publication. J-Y. Park and H.S. Lee are professional biostatisticians and were responsible for the analysis and interpretation of the data. J. Suh, J.Y. Song, M.K. Byun, J.H. Cho, H.J. Kim, J-H. Lee and J-W. Park collected and analysed the data, contributed to the drafting of the manuscript, revised the article, and approved the final version of this manuscript for publication. H.J. Park provided constructive criticism on the rationale and design of this study, interpreted the data, drafted and revised the article, and approved the final version of this manuscript for publication.

Conflict of interest: None declared.

\section{References}

1 Chen N, Zhou M, Dong X, et al. Epidemiological and clinical characteristics of 99 cases of 2019 novel coronavirus pneumonia in Wuhan, China: a descriptive study. Lancet 2020; 395: 507-513.

2 Huang C, Wang Y, Li X, et al. Clinical features of patients infected with 2019 novel coronavirus in Wuhan, China. Lancet 2020; 395: 497-506.

3 Wang $\mathrm{D}$, $\mathrm{Hu} \mathrm{B}$, $\mathrm{Hu} \mathrm{C}$, et al. Clinical characteristics of 138 hospitalized patients with 2019 novel coronavirus-infected pneumonia in Wuhan, China. JAMA 2020; 323: 1061-1069. 
4 Georas SN, Rezaee F. Epithelial barrier function: at the front line of asthma immunology and allergic airway inflammation. J Allergy Clin Immunol 2014; 134: 509-520.

5 Makris S, Johnston S. Recent advances in understanding rhinovirus immunity. F1000Res 2018; 7: F1000.

6 Holgate ST. Innate and adaptive immune responses in asthma. Nat Med 2012; 18: 673-683.

7 Goyal P, Choi JJ, Pinheiro LC, et al. Clinical characteristics of covid-19 in New York City. N Engl J Med 2020; 382: 2372-2374.

8 Butler MW, O'Reilly A, Dunican EM, et al. Prevalence of comorbid asthma in COVID-19 patients. J Allergy Clin Immunol 2020; 146: 334-335.

9 Charlson ME, Pompei P, Ales KL, et al. A new method of classifying prognostic comorbidity in longitudinal studies: development and validation. J Chronic Dis 1987; 40: 373-383.

10 Choi JY, Yoon HK, Lee JH, et al. Current status of asthma care in South Korea: nationwide the Health Insurance Review and Assessment Service database. J Thorac Dis 2017; 9: 3208-3214.

11 Park HJ, Byun MK, Kim HJ, et al. Regular follow-up visits reduce the risk for asthma exacerbation requiring admission in Korean adults with asthma. Allergy Asthma Clin Immunol 2018; 14: 29.

12 Kim MH, Rhee CK, Shim JS, et al. Inhaled corticosteroids in asthma and the risk of pneumonia. Allergy Asthma Immunol Res 2019; 11: 795-805.

13 Lee E, Kim A, Ye Y-M, et al. Increasing prevalence and mortality of asthma with age in Korea, 2002-2015: a nationwide, population-based study. Allergy Asthma Immunol Res 2020; 12: 467-484.

14 Park SY, Kim JH, Kim HJ, et al. High prevalence of asthma in elderly women: findings from a Korean national health database and adult asthma cohort. Allergy Asthma Immunol Res 2018; 10: 387-396.

15 Kim SY, Jung JY, Park MS, et al. Increased prevalence of self-reported asthma among Korean adults: an analysis of KNHANES I and IV data. Lung 2013; 191: 281-288.

16 Zhang JJ, Dong X, Cao YY, et al. Clinical characteristics of 140 patients infected with SARS-CoV-2 in Wuhan, China. Allergy 2020; 75: 1730-1741.

17 Lupia T, Scabini S, Mornese Pinna S, et al. 2019 novel coronavirus (2019-nCoV) outbreak: a new challenge. J Glob Antimicrob Resist 2020; 21: 22-27.

18 Garg S, Kim L, Whitaker M, et al. Hospitalization rates and characteristics of patients hospitalized with laboratory-confirmed coronavirus disease 2019 - COVID-NET, 14 States, March 1-30, 2020. MMWR Morb Mortal Wkly Rep 2020; 69: 458-464.

19 Lee N, Allen Chan KC, Hui DS, et al. Effects of early corticosteroid treatment on plasma SARS-associated coronavirus RNA concentrations in adult patients. J Clin Virol 2004; 31: 304-309.

20 Alfaraj SH, Al-Tawfiq JA, Assiri AY, et al. Clinical predictors of mortality of Middle East Respiratory Syndrome Coronavirus (MERS-CoV) infection: a cohort study. Travel Med Infect Dis 2019; 29: 48-50.

21 Matsuyama S, Kawase M, Nao N, et al. The inhaled corticosteroid ciclesonide blocks coronavirus RNA replication by targeting viral NSP15. bioRxiv 2020; preprint [https://doi.org/10.1101/2020.03.11.987016].

22 Edagawa S, Kobayashi F, Kodama F, et al. Epidemiological features after emergency declaration in Hokkaido and report of 15 cases of COVID-19 including 3 cases requiring mechanical ventilation. Global Health Med 2020; 2 : $112-117$.

23 Del Río-Navarro B, Gazca-Aguilar A, Quibrera Matienzo JA, et al. Metabolic and electrocardiographic effects of albuterol in pediatric asthmatic patients treated in an emergency room setting. Allergol Immunopathol 1999; 27: $18-23$.

24 Woodward S, Mundorff M, Weng C, et al. Incidence of supraventricular tachycardia after inhaled short-acting beta agonist treatment in children. J Asthma 2020; in press [https://doi.org/10.1080/02770903.2019.1709867].

25 O'Byrne PM, FitzGerald JM, Bateman ED, et al. Inhaled combined budesonide-formoterol as needed in mild asthma. N Engl J Med 2018; 378: 1865-1876.

26 Beasley R, Holliday M, Reddel HK, et al. Controlled trial of budesonide-formoterol as needed for mild asthma. N Engl J Med 2019; 380: 2020-2030.

27 Xiao C, Puddicombe SM, Field S, et al. Defective epithelial barrier function in asthma. J Allergy Clin Immunol 2011; 128: 549-556.

28 Lambrecht BN, Hammad H. The airway epithelium in asthma. Nat Med 2012; 18: 684-692.

29 Barton LM, Duval EJ, Stroberg E, et al. COVID-19 autopsies, Oklahoma, USA. Am J Clin Pathol 2020; 153: $725-733$ 